\title{
LA PARTICIPACIÓN ACTIVA COMO BASE FUNDAMENTAL PARA LA MEJORA DEL LANZAMIENTO EN BALONCESTO DE FORMACIÓN
}

\author{
Enrique Ortega Toro ${ }^{i}$, Aurelio Olmedilla Zafra ${ }^{1}$ y David Cárdenas Vélez ${ }^{2}$ \\ Universidad Católica San Antonio de Murcia ${ }^{1}$, Universidad de Granada ${ }^{2}$ \\ España \\ E-mail: eortega@pdi.ucam.edu
}

\begin{abstract}
Resumen
Ortega-Toro, E.; Olmedilla-Zafra, A. y Cárdenas-Vélez, D. (2007). La participación activa como base fundamental para la mejora del lanzamiento en baloncesto de formación. Revista de Ciencias del Ejercicio y la Salud, 5(1), 1-8. Muchas son las variables psicológicas que influyen en el rendimiento deportivo en general y en la eficacia de acciones concretas en particular. El presente trabajo de investigación relaciona el nivel de autoeficacia del jugador con balón en baloncesto y el número y grado de eficacia de lanzamientos obtenido durante la competición, con el objetivo de poder establecer valores iniciales que ayuden a definir estilos y sistemas de juego adaptados a las características del joven jugador. Para la realización del presente trabajo se administró un cuestionario de autoeficacia específica al contexto y se analizaron todos los lanzamientos de un total de 22 partidos, con una muestra de 187 jugadores. Los resultados señalan que existe poco reparto en el número de lanzamientos a canasta, siendo unos pocos jugadores los que realizan la gran totalidad de lanzamientos. De igual forma se aprecia que los jugadores con mayores niveles de autoeficacia específica anotaron mayor número de puntos $(\mathrm{p} .=.009)$ y realizaron mayor número de lanzamientos en juego $(\mathrm{p} .=.018)$. Estos datos reflejan la necesidad de modificar los estilos y sistemas de juego utilizados en competición, así como fomentar los niveles de autoeficacia en los jóvenes jugadores de baloncesto, con el objetivo de incrementar en cantidad y calidad su práctica deportiva.
\end{abstract}

PALABRAS CLAVE: autoeficacia, baloncesto formación, lanzamiento.

\section{INTRODUCCIÓN}

Según la Real Academia Española (1998), participar significa "tomar uno parte en una cosa", lo cual llevado al campo de la actividad física $y$ el deporte en general supone tomar parte en el juego deportivo y/o actividad física determinada. En el deporte del baloncesto, los jugadores pueden participar desde muchas y diversas perspectivas. Dentro de la competición, un jugador puede participar dentro del campo, es decir, jugando, o bien estar actuando desde el banquillo, fundamentalmente dando ánimos y consejos a sus compañeros.

Está claro que el deportista progresa en los entrenamientos, pero aprende a competir en los partidos (Antón, 1997; Cruz, Boixados, Torregrosa y Mimbrero, 1996; Junoy, 1996; Ortega, Piñar y Cárdenas, 1999). Las experiencias prácticas que un jugador puede adquirir en un partido no pueden ser sustituidas por ninguna situación de las que se propongan en los entrenamientos. Aunque el entrenador realice el esfuerzo por conseguir la mayor similitud entre ambas, el carácter agonístico y motivante que intrínsecamente conlleva la competición no podrá ser reemplazado por ninguna tarea que se proponga en los entrenamientos (Alonso, Boixados y Cruz, 1995; Antón, 1999; Cárdenas, 2003; Gordillo, 1992; Junoy, 1996; Ortega, Piñar, Alarcón y Cárdenas, 2002; Saura, 1996). Por lo tanto la única forma de conseguir que un jugador consiga competir a un nivel aceptable será asegurarse de que a lo largo de su vida deportiva haya acumulado muchas y muy diversas experiencias de competición.

En el caso de que el jugador esté participando dentro del campo, estará actuando unas veces como defensor, otras como atacante sin balón y otras como atacante con balón, siendo esta última situación necesaria e imprescindible para el proceso de formación del joven jugador de 
baloncesto en etapas de formación, ya que esta situación es considerada como la más motivante, además de ser necesaria para alcanzar el mayor logro posible $\mathrm{y}$, por lo tanto, la mayor satisfacción en el juego del baloncesto: el enceste (Cárdenas y Pintor, 2001; Giménez y Sáenz-López, 1999; Ortega et al., 1999).

Es obvio señalar que la principal forma que tiene el joven jugador de baloncesto de participar en la competición es a través de la práctica deportiva, es decir, se participa jugando, entendiéndose participación activa no sólo como estar jugando, sino como una participación que implica protagonismo y responsabilidad directa en el propio juego.

Todo ello puede llevar a pensar que el tiempo que cada jugador participe de forma activa en la competición tendrá una relación estrecha y directa con el grado de formación alcanzado (Comas, 1991; Devís, 1997; Giménez y Sáenz-López 1999; Ortega et al., 2002). En este sentido, la acción del lanzamiento es considerada como la acción definitoria $\mathrm{y}$ decisiva en el juego del baloncesto, por lo que formará pieza imprescindible del concepto de participación activa, es decir, para participar de forma activa en el juego, y que dicha competición sea un estímulo para la mejora del joven jugador, será necesario asegurar un mínimo de lanzamiento a canasta.

Dicho aumento en la diversidad de experiencias competitivas aportará, además de una mejora técnico-táctica, una mejora en el desarrollo psicológico del jugador, ayudándole a desarrollar su capacidad atencional y de concentración al proporcionarle experiencias que le ayuden a desarrollar y fortalecer su autoconfianza y autoeficacia, mejorando su autoconcepto y su autoestima, y aumentando su capacidad de autocontrol (Lozano, 2001).

\section{Hipótesis y Objetivos}

La hipótesis del presente trabajo es:

- El tipo de participación del jugador con balón está directamente relacionado con su nivel de Autoeficacia Específica, de manera que aquellos jugadores que disponen de valores más altos en las variables relacionadas con el lanzamiento, muestran niveles superiores de Autoeficacia Específica.

Los objetivos del presente trabajo son:

- Conocer las características del lanzamiento en baloncesto de formación.

- Analizar la posible relación entre la autoeficacia percibida y el lanzamiento en baloncesto de formación, tanto desde el punto de vista del número de lanzamientos realizados, como desde el punto de vista de su eficacia.

\section{MÉTODO}

Sujetos: la muestra objeto de estudio está formada por los jugadores de baloncesto participantes en la Fase Final del Campeonato de Andalucía Cadete (2000-2001) en el que participaron 16 equipos, con una muestra total de 187 jugadores.

Instrumentos y materiales: para evaluar el nivel de autoeficacia específica del jugador con balón en baloncesto, se ha utilizado el cuestionario elaborado por Ortega (2004) denominado "Cuestionario de Autoeficacia Específica del jugador con balón".

Procedimiento: para llevar a cabo el presente trabajo de investigación se siguieron las siguientes fases:

1- $\quad$ Filmación de un total de 24 partidos.

2- Elaboración de un sistema de categorías para estudiar el grado de participación del jugador con balón.

3- Administración del cuestionario. El cuestionario se administró al principio de la competición. Para rellenar dichos cuestionarios se citó a cada equipo por separado (solamente a los jugadores, no al equipo técnico) en un aula o salón del hotel en el cual estaban hospedados, con el objetivo de conseguir el clima adecuado que favoreciese la concentración. El cuestionario se administró a todos los equipos por la 
misma persona, para eliminar posibles variables contaminantes.

4- Entrenamiento de los observadores, para lo cual se siguió el protocolo elaborado por Behar (1993). Se realizó el entrenamiento de doce observadores hasta lograr coeficientes de fiabilidad inter e intra observador superiores a 0.95 .

5- Registro y análisis de los datos. Se registraron todos los lanzamientos de un total de 22 partidos. Una vez tomados los datos, se transcribieron al programa informático S.P.S.S. en la versión 11.5., y se analizaron los datos a través de análisis descriptivo e inferencial (ANOVA de un Factor con Post Hoc Scheffé).

Diseño: el trabajo de investigación se realizó a través de una metodología no experimental, descriptiva, transversal y correlacional (Hernández, Fernández y Baptista, 2000; Sierra-Bravo, 1999).

\section{RESULTADOS}

En la tabla 1, se aprecian los valores que Ortega (2004) recoge tras la validación y administración del cuestionario de participación del jugador con balón en baloncesto a veintidós jueces expertos. Estos jueces expertos señalan que para considerar la competición como un verdadero medio formativo, y que suponga un estímulo positivo para la mejora del jugador, debe realizar un número de lanzamientos en juego superior a 5, anotar más de 7 puntos y obtener una eficacia en el lanzamiento superior al $28.75 \%$. De igual modo, los jueces expertos señalan como muy adecuado para que la competición sea verdaderamente formativa realizar más de 10.5 lanzamientos, anotar más de 15 puntos y obtener un grado de eficacia superior al $49 \%$.

Tabla 1.- Valores medios, de las variables estudiadas, según el juicio de expertos (Tomado de Ortega, 2004).

\begin{tabular}{|c||c||c||c||c||}
\hline VARIABLE & NADA ADECUADO & $\begin{array}{c}\text { POCO } \\
\text { ADECUADO }\end{array}$ & $\begin{array}{c}\text { MEDIANAMENTE } \\
\text { ADECUADO }\end{array}$ & $\begin{array}{c}\text { MUY } \\
\text { ADECUADO }\end{array}$ \\
\hline \hline Lanzamientos en juego & 0 & $0.1-5$ & $5.01-10.5$ & +10.5 \\
\hline Puntos anotados & 0 & $0.1-7$ & $7.01-15$ & +15 \\
\hline $\begin{array}{c}\text { Porcentaje de eficacia en el } \\
\text { lanzamiento de 2 puntos }\end{array}$ & 0 & $0.1-28.75$ & $28.76-49$ & +49 \\
\hline
\end{tabular}

A partir de los valores registrados en la tabla 1 , en la tabla 2 se observa el número de jugadores que pertenecen a cada una de las categorías analizadas, en la que cabe destacar que:

- El $51.33 \%$ de los jugadores analizados no realizan el mínimo de lanzamientos en juego considerado como necesarios por los expertos para una adecuada formación, si bien, destaca que casi uno de cada cinco jugadores (19.25\%) realizan un número de lanzamientos en juego considerado como muy adecuado.

- Los jugadores que no logran ningún punto $(12.30 \%)$ sumados a los que consiguen anotar pocos puntos (53.48\%), representan un $65.78 \%$ del total. Esto significa que dos de cada tres jugadores no obtienen el mínimo de puntos por partido considerado como apropiado por los jueces expertos para una correcta formación. 
- El elevado porcentaje de jugadores que no obtienen ninguna eficacia en el lanzamiento de 2 puntos (19.25\%), que sumado al porcentaje de los jugadores con poca eficacia $(20.32 \%)$, permite afirmar que casi la mitad de los jugadores participantes en el campeonato analizado no disponen de un porcentaje de acierto en el lanzamiento de 2 puntos mínimo para un adecuado proceso de formación

Tabla 2.- Cantidad de jugadores, según su participación, en las categorías analizadas.

\begin{tabular}{|c||c|c|c||c||}
\hline VARIABLE & NADA ADECUADO & $\begin{array}{c}\text { POCO } \\
\text { ADECUADO }\end{array}$ & $\begin{array}{c}\text { MEDIANAMENTE } \\
\text { ADECUADO }\end{array}$ & $\begin{array}{c}\text { MUY } \\
\text { ADECUADO }\end{array}$ \\
\hline \hline Lanzamientos en juego & 14 & 82 & 55 & 36 \\
\hline Puntos anotados & 23 & 100 & 49 & 51 \\
\hline $\begin{array}{c}\text { Porcentaje de eficacia en el } \\
\text { lanzamiento de 2 puntos }\end{array}$ & 36 & 38 & 62 & 515 \\
\hline
\end{tabular}

En la tabla 3, se aprecia el mínimo, el máximo, la media y la desviación típica de los valores obtenidos tras administrar el cuestionario de "Autoeficacia específica del jugador con balón" a un total de 182 jugadores de baloncesto.

Tabla 3.- Valores descriptivos del “Cuestionario de Autoeficacia específica del jugador con balón”.

\begin{tabular}{|c|c|c|c|c|}
\hline $\mathrm{N}$ & MÍNIMO & MÁXIMO & MEDIA & DESV-TIP \\
\hline 187 & 93 & 295 & 222,72 & 34,650 \\
\hline
\end{tabular}

Posteriormente, y tras la realización de un análisis de frecuencias, se definieron tres grupos de jugadores: el grupo que disponía de poca autoeficacia ( $\begin{array}{lll}0 & \text { y } & 208\end{array}$ puntos), el grupo que disponía de una autoeficacia considerada como regular (209 y 237) y el grupo que disponía de mucha autoeficacia (238 hasta 310$)$.
A partir de estos datos, se calcularon las posibles diferencias entre estos tres grupos, con respecto a las variables relacionadas con el lanzamiento en juego.

A este respecto en la tabla 4 se observan las medias, por partido, de las variables relacionadas con el lanzamiento, de cada uno los tres grupos de jugadores, según el nivel de Autoeficacia específica del jugador con balón (poca-regular-mucha). 
Tabla 4.- Diferencias entre grupos, según los niveles de Autoeficacia Específica.

\begin{tabular}{||l|c|c|c|c||}
\hline \multicolumn{1}{||}{ VARIABLES DEL JUGADOR CON BALÓN } & \multicolumn{3}{c||}{ AUTOEFICACIA PERCIBIDA } \\
\hline & $\begin{array}{c}\text { POCA } \\
\mathrm{n}=62\end{array}$ & $\begin{array}{c}\text { REGULAR } \\
\mathrm{n}=62\end{array}$ & $\begin{array}{c}\text { MUCHA } \\
\mathrm{n}=63\end{array}$ & $\mathrm{p}$. \\
\hline Lanzamientos en juego & 4.74 & 6.64 & 7.33 & .018 \\
\hline Lanzamiento en carrera & 1.47 & 2.25 & 2.73 & .011 \\
\hline Lanzamiento en Salto & 3.19 & 4.31 & 4.58 & .073 \\
\hline Lanzamientos intentados de 1 punto & 1.45 & 2.30 & 2.41 & .027 \\
\hline Lanzamientos convertidos de 1 punto & 0.66 & 1.30 & 1.31 & .022 \\
\hline $\begin{array}{l}\text { Porcentaje de eficacia en el lanzamiento } \\
\text { de 1 punto }\end{array}$ & 29.80 & 38.59 & 40.51 & .130 \\
\hline Lanzamientos intentados de 2 puntos & 4.38 & 5.47 & 5.89 & .157 \\
\hline Lanzamientos convertidos de 2 puntos & 1.61 & 2.16 & 2.39 & .102 \\
\hline $\begin{array}{l}\text { Porcentaje de eficacia en el lanzamiento } \\
\text { de 2 puntos }\end{array}$ & 32.56 & 37.00 & 32.19 & .456 \\
\hline Lanzamientos intentados de 3 puntos & 0.36 & 1.17 & 1.45 & .002 \\
\hline Lanzamientos convertidos de 3 puntos & 0.09 & 0.21 & 0.37 & .004 \\
\hline $\begin{array}{l}\text { Porcentaje de eficacia en el lanzamiento } \\
\text { de 3 puntos }\end{array}$ & 8.34 & 9.71 & 15.67 & .128 \\
\hline Puntos anotados & 4.13 & 6.32 & 7.06 & .009 \\
\hline
\end{tabular}

Como se aprecia en la tabla 4 , en todas las variables objeto de estudio (salvo en el porcentaje de eficacia en el lanzamiento de 2 puntos) el grupo formado por los jugadores que obtienen mucha Autoeficacia Específica, muestra valores superiores al resto, apreciándose, en la mayoría de los casos, diferencias estadísticamente significativas. Estas diferencias se incrementan si se comparan únicamente el grupo que obtiene poca autoeficacia con el que tiene mucha.

En este sentido cabe destacar las diferencias estadísticamente significativas existentes en:

- El número de lanzamientos en juego $(p=.018)$, concretamente entre el grupo de jugadores con mucha autoeficacia y el grupo con poca $(\mathrm{p}=.021)$.

- El número de puntos anotados ( $\mathrm{p}=.009)$, en concreto entre el grupo de jugadores con poca autoeficacia y el grupo con regular $(\mathrm{p}=.044)$, así como entre el grupo con poca y el que muestra mucha $(p=.008)$.

\section{DISCUSIÓN}

En primer lugar, destaca el gran poder de predicción del cuestionario de Autoeficacia Específica del jugador con balón con respecto a las diferentes variables relacionadas con el lanzamiento, existiendo diferencias, estadísticamente significativas, entre aquellos jugadores que muestran mucha autoeficacia y los que disponen de poca; por lo tanto, aquellos jugadores que obtienen valores muy elevados de autoeficacia posteriormente en la competición son los que obtienen valores más elevados en las variables relacionadas con el lanzamiento en juego; en concreto, cabe destacar las diferencias significativas en el número puntos anotados, en los lanzamientos en juego y en los lanzamientos intentados y convertidos de 3 y 1 puntos; así como se aprecian diferencias aunque no sean significativas a favor del grupo de jugadores con mayor Autoeficacia Específica en relación con el número de lanzamientos intentados y convertidos de 2 puntos.

Desde una perspectiva general, estos datos coinciden con los de los estudios 
realizados sobre autoeficacia y ejecución y/o rendimiento por Lee (1982), utilizando aparatos gimnásticos, Barling y Abel (1983), en tenis, Lee (1989), en Hockey, Weiss Wiese y Klint (1989), con gimnastas, Deeter (1989), en una tarea de resistencia, Balaguer et al. (1990a, 1990b), en baloncesto, Martin y Gill (1991), con adolescentes varones corredores de fondo, Fitzsimmons, Landers, Thomas y Vander Mars (1991), con halterófilos, Miller, Carlyle y Pease (1992), en natación, hockey hielo y baloncesto, Yang y Pargman (1993), en karatekas, Lázaro y Villamarín (1993), con jugadoras de baloncesto, Palomares (1994), en una tarea atlética, Theodorakis (1995), con nadadores, Kane, Marks, Zaccaro y Blair (1996), en las prorrogas de los partidos, Theodorakis (1996), con tenistas, Guzmán (1996), en tareas atléticas, Kavussanu, Crews y Gill (1998), en una tarea de tiros libres en baloncesto, Rogers (1998), en kayak, Lu (1998), en bádminton, Psychountaki y Zervas (2000), con nadadores, Moritz, Feltz, Fahrback y Mack (2000), a través de un meta-análisis de 120 estudios, Bund (2001), en ciclismo, Treasure, Monson y Lox (2001), en tareas de fuerza y Sánchez y Torregrosa (2003), con escaladores, en los que se señala que la relación entre ambas es muy positiva, siendo la primera una gran predictora de la segunda.

A partir del presente estudio, nos atrevemos a señalar que el que un jugador participe de forma activa en el juego (con un número de lanzamientos elevado) puede revertir en un elevado nivel de Autoeficacia Específica, necesario para percibir la competición como un reto superable, disfrutar con la actividad deportiva propiamente dicha, emplear mayor esfuerzo ante las dificultades $\mathrm{y}$, en el caso de no superarla (perder en la competición), recuperar la percepción de eficacia de manera rápida. No obstante, el papel que juega en este aspecto el entrenador, y en el mejor de los casos el psicólogo deportivo, será determinante.

Así pues, uno de los objetivos que cualquier docente, ya sea profesor, como entrenador debe plantearse, es lograr incrementar los niveles de autoeficacia en sus educandos/jugadores, a través de diferentes y variadas formas (Ortega, 2004)..

Desde la perspectiva de la competición, una de las herramientas fundamentales de las que disponen los entrenadores para poder desarrollar eficazmente la autoeficacia, es el estilo de juego que, a su vez, incluye los sistemas de juego. Éstos deberán favorecer la adquisición de la mayor cantidad de experiencias posibles, y que dichas experiencias sean lo más variadas posible, con el objetivo de facilitar $\mathrm{y}$ enriquecer el proceso de aprendizaje del joven jugador.

Sin embargo, los datos reflejan todo lo contrario ya que se aprecia la cifra de jugadores que realizan un número de lanzamientos considerado por los expertos como adecuado es muy escaso, y aquellos que lanzan tienden a realizarlo siempre de la misma forma y en las mismas circunstancias; funciones previamente establecidas a través de la especialización temprana en el desempeño de puestos específicos, observándose una gran tendencia a entrenar y jugar con modelos muy semejantes a los utilizados por equipos senior, e incluso profesionales. Esto permite afirmar que en lugar de adaptar el juego según las características del joven jugador, se está adaptando el jugador al juego, lo que nos lleva a pensar que se considera al joven jugador, no como un adolescente en proceso de formación, sino como un adulto "en pequeño".

Por lo tanto, será necesario diseñar estilos de juego y aplicar modificaciones reglamentarias que fomentan que la gran mayoría de jugadores tengan la posibilidad de experimentar un número de lanzamientos en juego suficientes para considerar la competición como un verdadero medio formativo.

\section{REFERENCIAS}

Alonso, C., Boixados, M. y Cruz, J. (1995). Asesoramiento a entrenadores de baloncesto: efectos de la motivación deportiva en los 
jugadores. Revista de Psicología del Deporte, 7-8, 135-146.

Antón, J.L. (1997). Apuntes del I Curso sobre entrenamiento deportivo en edad escolar. Málaga: Instituto Andaluz del Deporte.

Antón, J.L. (1999). Valoración, importancia y formas de tratamiento y organización de la competición en el aprendizaje del balonmano. En J. L. Antón y L. J. Chirosa (Eds.), Jornadas de formación en balonmano: Alternativas y factores a valorar en el aprendizaje (pp.5-24). Granada: Editores.

Balaguer, I., Colilla, A, Gimeno, C. y Soler, M.J. (1990a). Diferencias sexuales en autoeficacia física, autoconfianza y ansiedad en jugadores de baloncesto. En I. Balaguer (Ed.), III Congreso Nacional de Psicología Social. Libro de comunicaciones. Volumen II (pp.484-489). Alicante: Editor.

Balaguer, I., Colilla, A., Gimeno, C. y Soler, M.J. (1990b). Influencia de la Autoeficacia, la ansiedad y la autoconfianza sobre la ejecución de los jugadores de baloncesto. III Congreso Nacional de Psicología Social. Libro de comunicaciones. Volumen II (pp.473-483). Alicante: Editor.

Barling, J. y Abel, M. (1983). Self-efficacy beliefs and tennis Performance. Cognitive Therapy and Research 7 (3), 265-272.

Bund, A. (2001). Zur Bedeutung des allgemeinen und aufgabenbezogenen Selbstvertrauens fuer das Bewegungslernen. Psychologie und Sport Schorndorf, 8(3), 78-90.

Cárdenas, D. (2003). El proceso de formación táctica colectiva desde una perspectiva constructivista. En A. López, C. Jiménez y R. Aguado (Eds.), Didáctica del baloncesto en las etapas de formación (pp.179-209). Madrid: Editores.

Cárdenas, D. y Pintor, D. (2001). La iniciación al baloncesto en el medio escolar. En F. Ruiz, A. García y A.J. Casimiro. (Eds.), La iniciación deportiva basada en los deportes colectivos: nuevas tendencias metodológicas (pp.105144). Madrid: Gymnos.

Comas, M. (1991). Estadísticas y su utilidad. La tecnología al servicio del baloncesto. Madrid: Gymnos.

Cruz, J., Boixados, M., Torregrosa, M. y Mimbrero, J. (1996). ¿Existe un deporte educativo?: Papel de las competiciones deportivas en el proceso de socialización del niño. Revista de Psicología del deporte, 9-10, 111-132.
Deeter, T.E. (1989). Re-modeling expectancy and value in physical activity. Journal of Sport and Exercise Psychology, 12(1), 86-91.

Devís, J. (1997). Educación física, deporte y curriculum. Investigación y desarrollo curricular. Madrid: Visor Distribuciones, S.A.

Fitzsimmons, P.A., Landers, D.M., Thomas, J.R. y Vander Mars, H. (1991). Does Self-efficacy predict Performance in experienced weightlifters?. Research Quarterly for Exercise and Sport, 62(4), 424-431.

Giménez, F.J. y Sáenz-López, P. (1999). Aspectos teóricos y prácticos de la iniciación al baloncesto. Huelva: Diputación de Huelva.

Gordillo, A. (1992). Orientaciones psicológicas en la iniciación deportiva. Revista de Psicología del Deporte 1, 27-36.

Guzmán, J.F. (1996). Análisis de la teoría de la autoeficacia en una tarea atlética. [Tesis Doctoral]. Valencia. Universidad de Valencia.

Hernández, R., Fernández, C. y Baptista, L. (2000). Metodología de la investigación ( ${ }^{a}$ Edición ampliada). México: MC Graw Hill.

Junoy, J. (1996). La actividad física y deportiva extraescolar en los centros educativos: Baloncesto. Madrid: Ministerio de Educación y Cultura.

Kane, T.D., Marks, M.A., Zaccaro, S.J. y Blair, V. (1996). Self-efficacy, personal goals, and wrestlers self-regulation. Journal of Sport and Exercise Psychology. 18(1), 36-48.

Kavussanu, M., Crews, D. y Gill, D. (1998). The effects of single versus multiple measures of biofeedback on basketball free throw shooting performance. International Journal of Sport Psychology 29(2), 132-144.

Lázaro, I. y Villamarín, F. (1993). Capacidad predictiva de la autoeficacia individual y colectiva sobre el rendimiento en jugadores de baloncesto. Revista de Psicología del Deporte, 4, 27-38.

Lee, C. (1982). Self-efficacy as a predictor of performance in competitive gymnastics. Journal of Sport Psychology, 4, 405-408.

Lee, C. (1989). The relationships between goal setting, self-efficacy, and female field. International. Journal of Sport Psychology, 20(2), 147-161.

Lozano, F. J. (2001). Papel de la Psicología de la actividad física y el deporte en la iniciación deportiva. En T. Pelegrín y E.J. Garcés de la Fayos (Coord.), Encuentro profesional de Psicología del deporte (pp.45-66). Murcia: Ayuntamiento de Beniel. 
Lu, J.H. (1998). Self-efficacy, competition outcome, and causal attributions in sport DissertationAbstracts International: SectionB: The Sciences and Engineering, 59 (5-B), 2486.

Martin, J.J. y Gill, D.L. (1991). The relationships among competitive orientation, sportconfidence, self-efficacy, anxiety, and performance. Journal of Sport and Exercise Psychology, 13(2), 149-159.

Miller, M., Carlyle, S. y Pease, R. (1992). The relationship between motivation and selfefficacy in competitive athletes participating in swimming, ice hockey, and basketball. Journal of Sport Behavior, 15(3), 201-208.

Moritz, S.E., Feltz, D.L., Fahrback, K.R. y Mack, D.E. (2000). The relation of self-efficacy measures to sport performance: a meta-analytic review. Research Quarterly for Exercise and Sport, 71(3), 280-294

Ortega, E. (2004). Análisis de la participación del jugador con balón en etapas de formación en baloncesto (14-16 años) y su relación con la Autoeficacia. [Tesis doctoral]. Universidad de Granada.

Ortega, E. Piñar, M.I. y Cárdenas, D. (1999). El estilo de juego de los equipos de baloncesto en las etapas de formación. Granada: Ortega, Piñar y Cárdenas.

Ortega, E., Piñar, M.I., Alarcón, F. y Cárdenas, D. (2002). Propuesta del número de jugadores que deben componer un equipo de baloncesto de categoría de formación. En M. Hernández, E. Navarro y A. Lorenzo (Coord.), Libro de Actas del II Congreso de Ciencias del Deporte. Madrid: Coordinadores.

Palomares, J.A. (1994). La autoeficacia y el rendimiento motor en estudiantes de secundaria. [Tesis Doctoral]. Valencia. Universidad de Valencia.

Psychountaki, M y Zervas, Y. (2000). Competitive worries, sport confidence, and performance ratings for young swimmers. Perceptual and Motor Skills, 91(1), 87-94.
Real Academia Española de la Lengua (1998). Diccionario de la Lengua Española. Madrid: Espasa Calpe, S.A.

Rogers, K.B. (1998). Physical self-efficacy and competitive state anxiety: An examination of adult participation in a non-traditional sport. Dissertation Abstracts International. SectionB: The Sciences and Engineering, 58(10-B), 5667.

Sánchez, J. y Torregrosa, M. (2003). Aspectos psicológicos básicos para la escalada según los expertos. En S. Márquez (Ed.), Libro de actas del IX Congreso Nacional de Psicología de la Actividad física y el deporte Perspectiva Latina (pp.279-284). León: Editor.

Saura, J. (1996). El entrenador en el deporte escolar. Lleida: Institut d'Estudis Ilerdencs.

Sierra-Bravo, R. (1999). Tesis Doctorales y trabajos de investigación científica. Madrid: Paraninfo.

Theodorakis, Y. (1995). Effects of Self-efficacy, satisfaction and personal goals on swimming performance. Sport psychologist, 9(3), 245253.

Theodorakis, Y. (1996). The influence of goals, commitment, self-efficacy and selfsatisfaction on motor performance. Journal of Applied Sport Psychology, 8(2), 171-182.

Treasure, D.C., Monson, J. y Lox, C. L. (2001). Relationship between self-efficacy, wrestling performance, and affect prior to competition. The Sport Psychologist, 10 (1), 73-83.

Weiss, M.R., Wiese, D.M. y Klint, K.A. (1989). Head over heels with success: The relationship between self-efficacy and performance in competitive youth gymnastics. Journal of Sport and Exercise Psychology, 11, 444-451.

Yang, G., Pargman, D. (1993). An investigation of relationships among sport-confidence, selfefficacy, and competitive anxiety and their ability to predict performance on a karate skill test. In S. Serpa, (Ed.), VIII World Congress of Sport Psychology (pp.968-972). Lisboan: Editor.

\footnotetext{
${ }^{1}$ Dirección para correspondencia: Enrique Ortega Toro. Universidad Católica San Antonio. Facultad de Ciencias de la Salud, de la Actividad Física y el Deporte. Campus de los Jerónimos, s/n. 30.107 GUADALUPE (Murcia). ESPAÑA. Correo electrónico: eortega@pdi.ucam.edu
} 